\title{
THOMAS III. VON SALUZZO, Le livre du Chevalier errant
}

\section{Piero Andrea Martina}

\section{(2) OpenEdition Journals}

\section{Edizione digitale}

URL: https://journals.openedition.org/studifrancesi/44714

DOI: 10.4000/studifrancesi.44714

ISSN: 2421-5856

\section{Editore}

Rosenberg \& Sellier

\section{Edizione cartacea}

Data di pubblicazione: 1 août 2021

Paginazione: 350-351

ISSN: 0039-2944

\section{Notizia bibliografica digitale}

Piero Andrea Martina, «Tномаs III. von saluzzo, Le livre du Chevalier errant», Studi Francesi [Online], 194 (LXV | II) | 2021, online dal 06 septembre 2021, consultato il 14 octobre 2022. URL: http://

journals.openedition.org/studifrancesi/44714 ; DOI: https://doi.org/10.4000/studifrancesi.44714

Questo documento è stato generato automaticamente il 14 octobre 2022.

\section{(c)}

Creative Commons - Attribuzione - Non commerciale - Non opere derivate 4.0 Internazionale - CC BYNC-ND 4.0

https://creativecommons.org/licenses/by-nc-nd/4.0/ 


\title{
THOMAS III. VON SALUZZO, Le livre du Chevalier errant
}

\author{
Piero Andrea Martina
}

\section{NOTIZIA}

THOMAS III. VON SALUZZo, Le livre du Chevalier errant, herausgegeben von Robert Fajen, Wiesbaden, Reichert, 2019, «Imagines Medii Aevi» 48, lxxxii + 715 pp.

1 A Robert Fajen si doveva già un fondamentale volume sullo Chevalier errant di Tommaso III di Saluzzo - Die Lanze und die Feder: Untersuchungen zum Livre du Chevalier errant von Thomas III., Markgraf von Saluzzo, Wiesbaden, Reichert, 2003 - oltre ad alcuni importanti contributi più puntuali sullo stesso testo. È pubblicata ora la sua eccellente edizione di quest'opera importante e dal fascino obliquo, oggi conservata in due manoscritti: Paris, BnF, fr. 12559 (P) e Torino, BNU, L. V. 6 (T), danneggiato dall'incendio del 1904 ma leggibile per buona parte. L'edizione, sia detto subito, è un poco differente dal modello di edizione oggi dominante per i testi medievali, francesi, ma non soltanto: l'introduzione ha uno spiccato taglio storico-letterario e non tralascia alcuni problemi filologici, ma non presenta uno studio linguistico o metrico del testo. Poco male, in realtà, lasciare spazio a lavori futuri, soprattutto per un testo che richiederebbe un'attenzione specifica a tali problemi.

2 Del testo di Tommaso di Saluzzo esisteva una prima edizione procurata da Marvin J. WARD nel 1984, A Critical Edition of Thomas III, marquis of Saluzzo's Le Livre du Chevalier Errant, tesi di dottorato discussa alla University of North Carolina at Chapel Hill, inedita ma accessibile agli studiosi in numerose biblioteche europee; e il volume curato da Marco PICCAT, in cui il testo è allestito da Laura RAMELlo e tradotto da Enrica MARTINENGo: Il Libro del Cavaliere Errante (BnF ms. fr. 12559), Boves, Araba Fenice, 2008, sulla quale si veda la scheda di Anna Maria Finoli in "Studi Francesi" n 161, 2010, pp. 344-346. Ward proponeva una trascrizione di $P$ corretta quando ciò era possibile, ma senza troppe spiegazioni, sulla base di T; Piccat e Ramello optavano per una trascrizione del solo $P$. 
3 Dei due manoscritti conservati dello Chevalier errant, $P$ è un sontuoso volume prodotto probabilmente per Tommaso stesso, che rimane nelle mani della famiglia marchionale di Saluzzo almeno per alcuni decenni del Quattrocento: il testo è accidentato da alcuni problemi nella lingua e nella rima e da numerosissime irregolarità metriche nelle parti in versi, tanto da rendere difficile ritrovare talora lo schema del distico di ottosillabi; in $P$ si trovano inoltre alcuni episodi assenti da $T . T$ presenta un testo decisamente più regolare (più corretto? ma sull'opportunità di questo aggettivo torna Fajen nell'introduzione) sul piano linguistico e metrico. Del romanzo Vincenzo Malcarne, a fine Settecento, descrisse un altro manoscritto, contenente «correzioncelle, ed aggiunte» apparentemente «del medesimo [Marchese] il quale dopo d'aver fatto trascrivere il suo lavoro» continuò "probabilmente» nel suo lavoro di correzione. La testimonianza di Malcarne è un poco problematica, in quanto il manoscritto con correzioni sarebbe secondo lui $T$ e non un "altro codice meno elegante da lui avuto», cosa che in generale è spiegata con una confusione tra $T$ e il codice perduto, recante correzioni (forse) d'autore, noto come $M$. Da qui l'idea di Ward secondo cui $M$ sarebbe l'originale, da cui sarebbe stato copiato $P$; dopo rimaneggiamento d'autore, da $M$ ' sarebbe stata tratta la copia $T$. Fajen mette in luce le debolezze del ragionamento di Ward e dunque i principi della sua costituzione del testo, decidendo di presentare il testo di $P$ corretto sulla base di $T$ soltanto in casi di evidente corruttela; in apparato, Fajen registra tutte le varianti di $T$. L'operazione è, di fatto, l'unica possibile; rimane il dubbio che $T$ offra effettivamente un testo migliore, ancorché materialmente lacunoso, che varrebbe la pena di leggere a stampa, anche date le condizioni di fragilità delle carte del manoscritto.

4 Il testo è stabilito con cura dall'editore. In corsivo nel testo si offre la descrizione delle miniature di $P$ mentre in apparato si registrano anche le rubriche di $T$, che in Ward si leggevano in una Table of contents che precedeva il testo. Utile per il lettore segnalare un Riassunto 'critico' del romanzo (pp.614-646), necessario per orientarsi nell'oceano narrativo di Tommaso, ma pure per ritrovare la segnalazione delle fonti.

5 Nell'introduzione Fajen riprende alcuni punti essenziali del suo studio del 2003: si dipinge il contesto storico necessario per comprendere l'autorappresentazione romanzesca di Tommaso e per collocare la sua attività letteraria, che è l'oggetto di un secondo, articolato, paragrafo. Fajen, ricordiamo, è sostenitore di una datazione alta del testo: prestando fede a quanto dichiarato dall'autore stesso, lo studioso colloca la sua composizione durante la prigionia torinese (1394-1396) successiva alla cattura nella battaglia di Monasterolo, senza sopravvalutare l'importanza del suo soggiorno parigino (1403-1405). La biografia è aggiornata rispetto allo studio (si potrebbe forse aggiungere: M. L. MENEGHETTI, Storie al muro, Torino, Einaudi, 2015).

6 Chiude l'edizione un glossario, un'utile citazione estensiva delle fonti letterarie dello Chevalier errant (nel testo delle edizioni moderne) messe a confronto con il testo di Tommaso, e l'indice dei nomi.

7 Il testo di Tommaso di Saluzzo ispiratore degli affreschi commissionati alla Manta dal figlio illegittimo Valerano, reggente del marchesato, continua ad affascinare, anche per le questioni che lascia aperte: la lingua del poema e dei manoscritti, i rapporti tra i manoscritti $P$ e $T$, la loro opaca storia quattrocentesca, la conoscenza della letteratura francese più recente da parte dell'autore. Lo studioso e il lettore interessato dispongono ora di un testo stabilito con attenzione nell'elegante volume di R. Fajen. 